\title{
The All-pairs Suffix-Prefix Matching Problem
}

\author{
William H. A. Tustumi (IC), Guilherme P. Telles (PQ), Felipe A. Louza (PG)
}

\section{Abstract}

The all-pairs suffix-prefix matching is a very important problem in string processing. Different solutions have been proposed to this problem. We present a new and improved algorithm that is 2.6 times faster and uses $15 \%$ less memory than the previous best know solution.

Key words: Suffix-prefix matching, Suffix array, LCP array

\section{Introduction}

The all-pairs suffix-prefix matching (APSP) is an important problem in string processing having application in the context of DNA sequencing. Given a set of $k$ strings $\{S 1, S 2, \ldots, S k\}$, the APSP is the problem of finding, for all pairs $\mathrm{Si}$ and $\mathrm{Sj}$, the longest suffix of $\mathrm{Si}$ that is a prefix of $\mathrm{Sj}$.

This problem has been solved optimally by Gusfield et al. ${ }^{1}$ in 1992 (using suffix trees) and almost 20 years later by Ohlebusch Gog $^{2}$ in 2010 (using enhanced suffix arrays). The later is about 3 times faster than the one that uses suffix trees.

\section{Results and Discussion}

We propose a new optimal algorithm that is faster and more space-efficient - in practice. Our algorithm scans the enhanced suffix arrays in a different way and uses a different auxiliary data structure.

The algorithm was implemented in $\mathrm{C}++$ using sdsl-lite library ${ }^{3}$. We used real DNA sequences of the EST database from C. elegans. We compared the performance of our algorithm with the algorithm $\mathrm{OG}^{2}$, best know solution.

Image 1 shows the total running time (in seconds) of each algorithm.

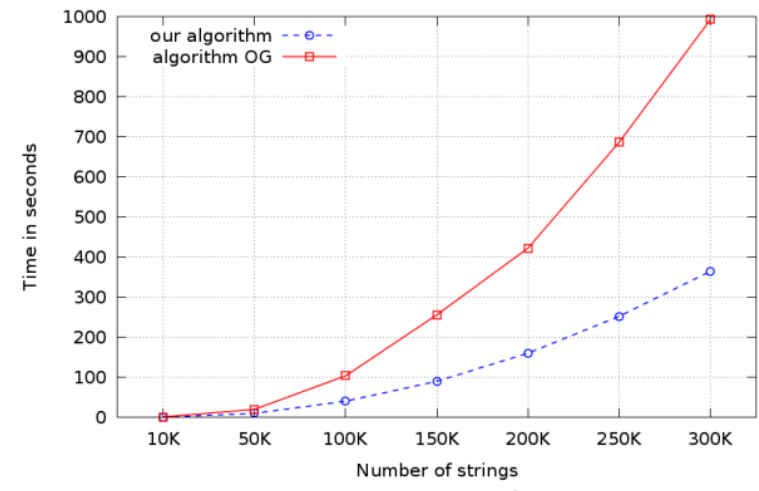

Image 1. Running time performed by each algorithm.
Image 2 shows the amount of memory used by each algorithm.

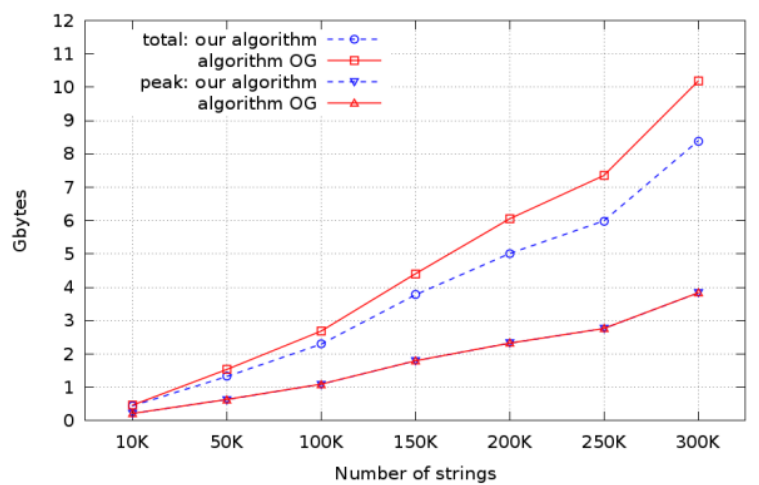

Image 2. Memory consumption of each algorithm.

One can see that our algorithm have outperformed algorithm OG by a factor of 2.6 on the average, and the total memory used by our algorithm was $15 \%$ less on average

\section{Conclusions}

We presented a faster and more space-efficient algorithm to solve the APSP. Our algorithm can be easily parallelized and modified to work in semi-external fashion.

\section{Acknowledgement}

The project was financed by CNPQ (grant No 118372/2014-9).

\footnotetext{
${ }^{1}$ Gusfield, D.; Landau, G. M.; Schieber, B.; (1992). An efficient algorithm for the all pairs suffix-prefix problem. Information Processing Letters, v. 41, n. 4, p.181-185.
}

${ }^{2}$ Ohlebusch, E.; Gog, S.; (2010). Efficient algorithms for the all-pairs suffix-prefix problem and the all-pairs substring-prefix problem. Information Processing Letters, v. 110 n. 3, p. 123-128.

${ }^{3} \mathrm{Gog}$, Simon, Timo Beller, Alistair Moffat, and Matthias Petri (2010)"From theory to practice: Plug and play with succinct data structures." In Experimental Algorithms, pp. 326-337.. 